\title{
GESTÃO À VISTA NA PRÁTICA EM EDUCAÇÃO PROFISSIONAL E TECNOLÓGICA: UMA APLICAÇÃO NA FACULDADE DE TECNOLOGIA SENAI/SC FLORIANÓPOLIS
}

Juliano Anderson Pacheco ${ }^{1}$ Maycon $\mathrm{Cim}^{2}$

\section{Resumo}

A comunicação é essencial nas empresas e um dos principais fatores para se construir uma liderança sólida. Dentro desse contexto, a gestão visual, quando bem desenvolvida, é uma prática eficaz e eficiente de comunicação. Este artigo tem como objetivo propor a implementação de gestão à vista em uma instituição de Educação Profissionale Tecnológica(EPT), através daidentificação de necessidades, elaboração e implantação de um mural de gestão à vista em uma faculdade de tecnologia do SENAI/SC. O referencial teórico desenvolvido nesta pesquisa tem início com a gestão estratégica, que visa aos resultados por meio de metas, passa pela ferramenta de gestão Plan-Do-Check-Act (PDCA) e finaliza com a abordagem da gestão à vista preconizada pelo Sistema Toyota de Produção. Metodologicamente, este trabalho é de natureza aplicada, com abordagem qualitativa e quantitativa; tem objetivo exploratório e caráter experimental, com confirmação por uma pesquisa-ação. As etapas desenvolvidas na metodologia foram o planejamento, a elaboração e a operacionalização do painel, a avaliação da implementação. Os primeiros resultados apontaram para um aumento do conhecimento de toda a equipe dos projetos existentes na Faculdade, pressupondo-se uma maior motivação na solução dos problemas que surjam em tais projetos. Com o conhecimento das agendas dos cursos e das pessoas, foi possível ter uma visão sistêmica dos recursos da Faculdade, tanto da equipe como dos ambientes educacionais. As necessidades essenciais na gestão de uma faculdade foram identificadas e a proposição de mural atendeu a faculdade de tecnologia. Percebeu-se, também, um aumento no compartilhamento de boas práticas entre os coordenadores de curso.

Palavras-Chave: TI - Gestão à Vista. Educação Profissional e Tecnológica. Metas. Indicadores. 


\section{INTRODUÇÃO}

A gestão profissional de negócios visa ao uso de ferramentas adequadas pelos gestores, de preferência em conjunto com a equipe, para facilitar o fluxo contínuo de informações e proporcionar o alcance das metas das organizações. Entende-se que a gestão visual é uma técnica que envolve e sensibiliza a equipe no conhecimento de informações relevantes para a execução de tarefas. As metas e os indicadores dos projetos, bem como o conhecimento dos focos de atuação e a expertise da equipe, são alguns exemplos de informações relevantes. $\mathrm{O}$ intuito é proporcionar um contexto favorável para as ações dos gestores e possibilitar facilidades para o alcance das metas das instituições.

Partindo da premissa de que a comunicação é um dos principais fatores que promovem um contexto adequável à gestão, definiu-se o seguinte problema de pesquisa:"De que forma a gestão à vista pode trazer benefícios na gerência de uma instituição de educação profissional ao ser implementada?"

Para resolver esse problema, o presente trabalho tem como objetivo propor a prática da gestão à vista em uma instituição de Educação Profissional e Tecnológica (EPT). Para atingir esse objetivo geral,definiram-se como objetivos específicos: (i) identificar as necessidades de práticas de gestão à vista aplicáveis à EPT; (ii) elaborar uma proposta de mural com painéis de gestão à vista para uma instituição de EPT; (iii) implantar a proposta de mural de gestão à vista em uma faculdade de tecnologia do SENAI/SC.

\section{GESTÃO ESTRATÉGICA, METAS E INDICADORES}

O projeto de gestão de uma empresa deve ser desenvolvido a fim de que as metas propostas sejam alcançadas pelos gestores, partindo de um pressuposto de que metas existem em todas as empresas.Algumas companhias possuem metas explícitas e estruturadas, como as que utilizam o Balanced Scorecard (BSC) (KAPLAN; NORTON, 2000); em contrapartida, existem empresas que possuem metas implícitas e desestruturadas, que se resumem, geralmente, em gerar lucros para os proprietários.

O referencial teórico deste trabalho trouxe os seguintes pontos para discussão: a gestão estratégica com vistas aos resultados por meio de metas (KAPLAN; NORTON, 2000); o uso da ferramenta de gestão Plan-Do-CheckAct (PDCA) - em português ficaria PlanejarFazer-Checar-Atuar - que permite o acompanhamento dos indicadores das metas propostas (FALCONI, 2009); e a ferramenta de Gestão à Vista, como uma forma que possibilita facilidade de comunicação com partes interessadas aos projetos da empresa (JONES, 2011). Tais ferramentas, PDCA e Gestão à Vista, são preconizadas pelo Sistema Toyota de Produção. (SOBEK; SMALLEY, 2010).

As organizações que possuem estratégias e se guiam por estas, segundo Kaplan e Norton (2000), devem seguir alguns princípios básicos: desdobrar a estratégia em um processo contínuo 
e como parte integrante da tarefa de todos, mobilizar a liderança como agente desse desdobramento, traduzindo a estratégia em ações operacionais e, assim, alinhar a organização às estratégias.

Para a operacionalização desses princípios, a ferramenta mais utilizada é o Balanced Scorecard (BSC), que organiza as estratégias em perspectivas, conforme representado na figura 1 . (KAPLAN; NORTON, 1997).

Figura 1: Perspectivas do BSC

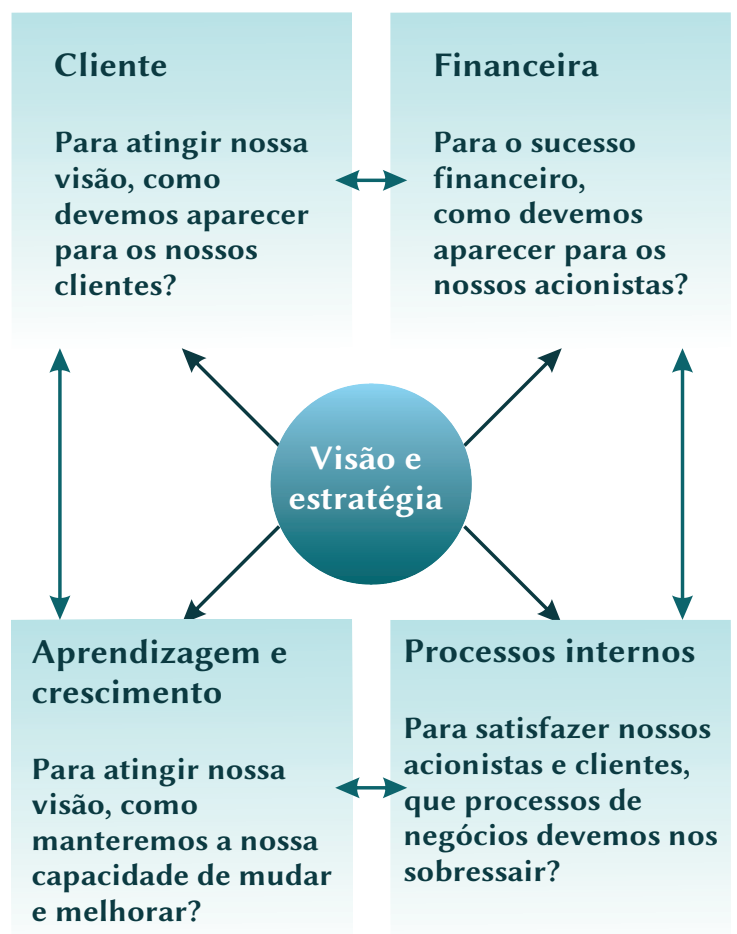

Fonte: BSI (2013)

As perspectivas propostas por Kaplan e Norton (2004) são: Financeira (Financial); Cliente (Customer); Processos Internos (Internal Business Processes); Aprendizado e Crescimento (Learning and Growth).Junto a tais perspectivas, são definidos objetivos estratégicos, metas e indicadores.

Na prática, o BSC se transformou em um sistema gerencial essencial, sendo utilizado como a principal ferramenta organizacional para mensuração dos processos gerenciais que desdobram as estratégias das organizações. Com o BSC, estabelece-se metas individuais e da equipe, planejamento, orçamentos com definição clara das metas e dos indicadores que representam as estratégias. (KAPLAN; NORTON, 1997).

\subsection{Ferramenta PDCA}

A base do PDCA está calcada na liderança, que segundo Falconi (2009,p.14),"precisa conseguir resultados por meio das pessoas, então a pessoa do líder deve investir uma parte substancial de seu tempo no desenvolvimento de sua equipe”. $\mathrm{O}$ autor defende que é necessário desenvolver a cultura da tomada de decisões baseada em fatos e dados, por meio do enfrentamento dos fatos, independente dos resultados serem bons ou ruins, pois se acredita que somente com o conhecimento necessário as situações adversas são reversíveis.

O PDCA consiste em um método gerencial, concernente à resolução de problemas, que de forma considerada simples, busca corrigir desvios das metas por meio de um processo sistemático de análise de indicadores e ações apropriadas sobre as causas desses problemas, para o retorno ao curso das metas. Para Falconi (2009, p. 25):

\section{Esse método viabiliza o Gerenciamento Científico da organi- zação. Veremos que o PDCA permite criar, aprender, copiar e difundir conhecimento e que o aprendizado é a alma da sua utilização. O PDCA transforma uma organização numa escola, pois a busca por resultados é paralela à busca do conhecimento.}

$\mathrm{Na}$ utilização prática, o PDCA consiste em um processo de busca para consolidação da padronização e aumento da qualidade, com foco na melhoria contínua ao longo do tempo, conforme representa a figura 2 . 
Figura 2: Processo do PDCA para a melhoria contínua

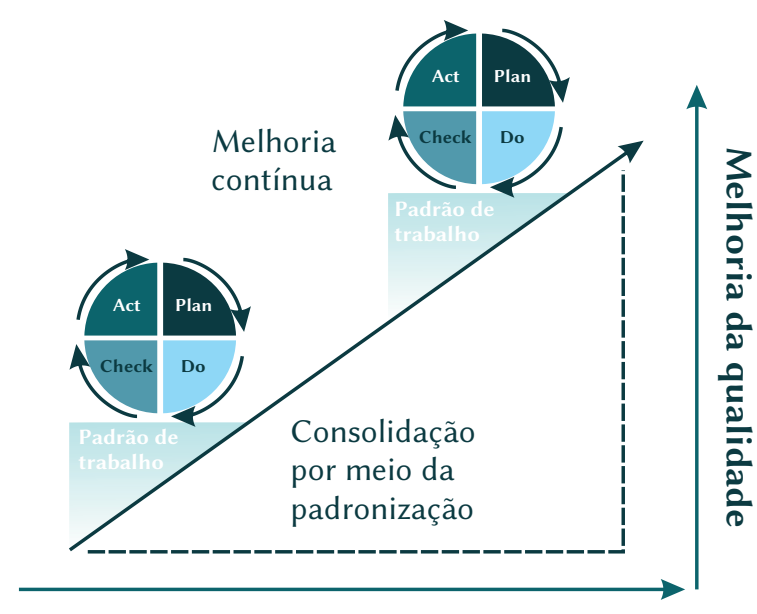

Tempo

Fonte: Wikimedia Commons (2014)

Percebe-se que o PDCA proporciona uma forma eficaz de realizar o BSC, pois promove ações que podem levar ao alinhamento entre as metas e os indicadores propostos no BSC. Conclui-se, assim, que com o uso efetivo do PDCA, o desdobramento das estratégias na operação é possível se for realizado de forma sistemática e com constância de propósito.

\subsection{Gestão Visual}

No Sistema Toyota de Produção Enxuta, uma das técnicas utilizadas amplamente é a gestão à vista, que segundo Jones (2011, p. 01):

'Aprender a enxergar' é o passo inicial para 'aprender a agir'. Fazendo com que os fatos de qualquer situação fiquem claramente visíveis, é muito mais fácil chegar a um consenso sobre o que é necessário ser feito, criar um compromisso em fazê-lo e manter o foco para sustentá-lo por muito tempo.
Para Jones (2011), a gestão visual permite o desdobramento da estratégia em escolhas visíveis, possibilitando a priorização de atividades e permitindo um diálogo eficaz com as equipes das organizações para operacionalizar as estratégias no nível operacional.

Empresas de classe mundial utilizam a gestão à vista nos seus ambientes como, por exemplo, a Boeing (TANNER, 2010) e a própria Toyota. (TANAKA, 2010). A figura 3 mostra como a Boeing faz uso da gestão à vista em seus projetos de engenharia junto a suas equipes técnicas de pesquisa e desenvolvimento de soluções para a indústria aeronáutica.

Figura 3: Gestão à vista na Boeing

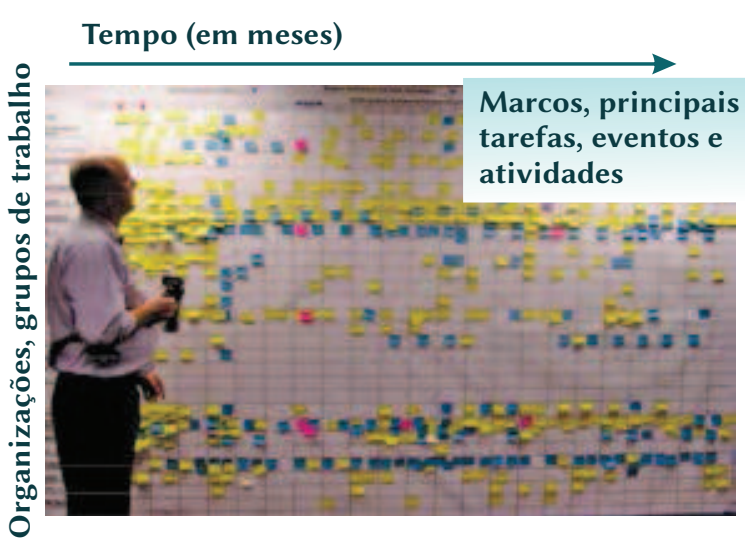

Fonte: Tanner (2010)

Para Shook (2012), os benefícios de tornar visuais os indicadores do trabalho, tendem a facilitar a resolução de problemas e a sustentação de ganhos, mas para isso, é necessário organizar o ambiente físico de forma adequada para esse fim, de maneira que a equipe se sinta motivada para o trabalho. Esse mesmo autor alerta que a proposição de tornar visuais indicadores de forma aleatória acarretará em resultados aleatórios e, portanto, em consequências geralmente 
indesejadas.Para Shook, é necessário organizar "o fluxo de trabalho para que os problemas sejam destacados conforme eles aparecem, capacitando e incentivando pessoas e equipes a enfrentá-los da forma certa”. (SHOOK, 2012, p. 07).

A partir do referencial teórico apresentado, identifica-se que, ao utilizar a gestão à vista, é possível comunicar de forma mais efetiva as estratégias da empresa.

Em linhas gerais, o BSC desdobra a estratégia em indicadores, que são acompanhados pelo PDCA e, quando observados pelas equipes por meio da gestão à vista, aumentam a possibilidade da estratégia ser operacionalizada.

\section{METODOLOGIA}

Para o desenvolvimento deste artigo, utilizou-se a pesquisa científica, que de forma organizada, permite provar ou não os objetivos e hipóteses inicialmente propostos pelo pesquisador. Para Severino (2007, p. 32): "a ciência se faz quando o pesquisador aborda os fenômenos aplicando recursos técnicos, seguindo um método e apoiando-se em fundamentos epistemológicos".

Este trabalho busca direcionar os conhecimentos a uma aplicação para a solução de um problema específico, e, portanto, é considerado de natureza aplicada. A forma de abordagem é qualitativa, pois busca a interpretação dos fenômenos e a atribuição de significados, e também é quantitativa, pois busca a quantização de fenômenos com uso de recursos e de técnicas estatísticas. A pesquisa tem objetivo exploratório para proporcionar maior familiaridade com o problema, visando a torná-lo explícito ou a construir hipóteses em torno dele. (SILVA; MENEZES, 2005).

Os autores Silva e Menezes também classificam a pesquisa com relação aos procedimentos técnicos. Considera-se que essa pesquisa é de caráter experimental, pois uma vez determinado um objeto de estudo, buscam-se variáveis que seriam capazes de influenciá-lo, as formas de controle e de observação dos efeitos que essas variáveis produzem no objeto. É também uma pesquisa do tipo pesquisa-ação, pois busca resolver um problema coletivo de forma participativa, com atuação do pesquisador e dos participantes que representam o problema.

Para Gil, a pesquisa "é desenvolvida mediante o concurso dos conhecimentos disponíveis e a utilização cuidadosa de métodos, técnicas e outros procedimentos científicos" (2002, p. 20). A forma organizada é composta de etapas que, segundo uma proposição de Gil (2002), pode ser esquematizada em 10 etapas. Para este trabalho, identificou-se sete etapas, conforme figura 4, pois não foi realizada, pelo autor, a coleta de dados primários para a elaboração da aplicação prática. 
Figura 4: Etapas da pesquisa científica utilizadas neste trabalho

\section{Formulação do problema}

Proposta de gestão à vista em uma Instituição de Educação Profissional

\section{Construção de hipóteses}

Gestão visual proporciona benefícios

\section{Determinação do plano}

Planejamento de um painel de gestão à vista

\section{Operacionalização das variáveis}

Elaboração de um painel de gestão à vista

\section{Coleta de dados \\ Operacionalização de um painel de gestão à vista \\ 9. Análise e interpretação dos resultados}

Avaliação da implantação do painel de gestão à vista

\section{Redação do relatório da pesquisa}

Registro do trabalho
As etapas subsequentes, desenvolvidas na metodologia, foram: o planejamento do painel, a elaboração e a operacionalização do mesmo, a avaliação da implementação e o registro do trabalho desenvolvido. Entende-se que essas etapas consistem nos resultados discutidos neste trabalho.

\section{RESULTADOS OBTIDOS}

Neste item estão apresentadas as etapas desenvolvidas para a implantação da gestão à vista, que consistem no planejamento, elaboração, operacionalização e avaliação dos primeiros resultados obtidos. No planejamento, apresenta-se a identificação das necessidades de informação para uma gestão eficaz. Na elaboração, é apresentada a forma de construção do painel, levando-se em consideração a sua forma de preenchimento. A operacionalização consiste nas ações de preenchimento do painel, bem como o envolvimento da equipe nesta etapa.
Finalmente, é apresentada a avaliação de todo processo de implantação da gestão à vista.

\subsection{Identificação das Necessidades de Gestão à Vista}

$\mathrm{Na}$ definição das necessidades, utilizaram-se algumas premissas norteadoras para facilitar a comunicação dos principais aspectos na gestão de um núcleo de negócio em EPT no SENAI/ SC. Considerou-se que o SENAI/SC é uma 
instituição que organiza o negócio Educação em projetos, que os principais recursos necessários na execução de cursos de EPT são docentes e ambientes de ensino e, por último, que o núcleo em questão é uma Faculdade de Tecnologia (Tecnólogos e Pós Lato Sensu). Diante dessas premissas, identificou-se que a estrutura adequada do painel proposto seja a apresentada na figura 5 .

Figura 5: Painel com as principais necessidades identificadas

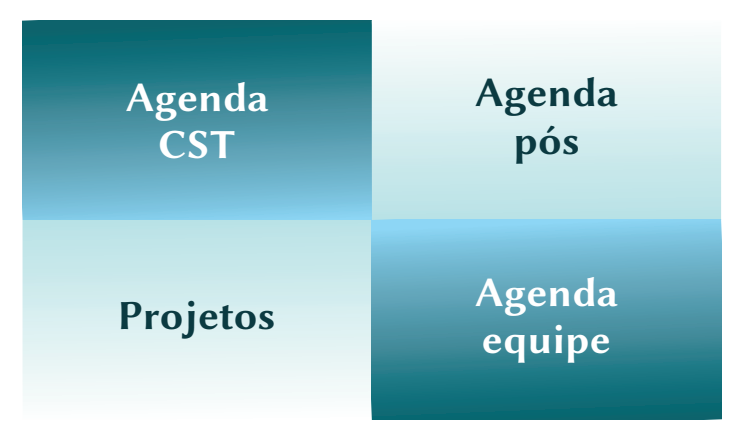

Fonte: Dos autores (2013)

Com base na figura 5, foram identificados quatro focos da Faculdade a serem organizadas em painéis:

- PROJETOS: informações das coordenações, principais metas e indicadores financeiros e de produção dos projetos do núcleo;
- AGENDA CST: horários dos Cursos Superiores de Tecnologia (CST), com as informações dos docentes em cada unidade curricular e respectivos ambientes;

- AGENDAPÓS: cronograma das aulas de pós com as informações dos docentes em cada unidade curricular e datas agendadas;

- AGENDA EQUIPE: ocupação semanal da equipe (coordenadores e docentes) com as informações do projeto e respectiva atividade.

Entende-se que,com a disseminação sistemática e permanente de informações sobre os projetos de educação, agenda dos cursos e da equipe, o conhecimento, é ampliado promovendo a comunicação eficaz das metas e dos indicadores entre todas as partes interessadas da Faculdade, desdobrando no nível tático-operacional.

\subsection{Elaboração dos Painéis de Gestão à Vista}

$\mathrm{Na}$ elaboração do painel, utilizou-se das premissas: custo, simplicidade e replicabilidade, buscando-se estruturar um painel de fácil elaboração com materiais simples e de fácil acesso. Os materiais escolhidos para o painel estão apresentados na figura 6. 


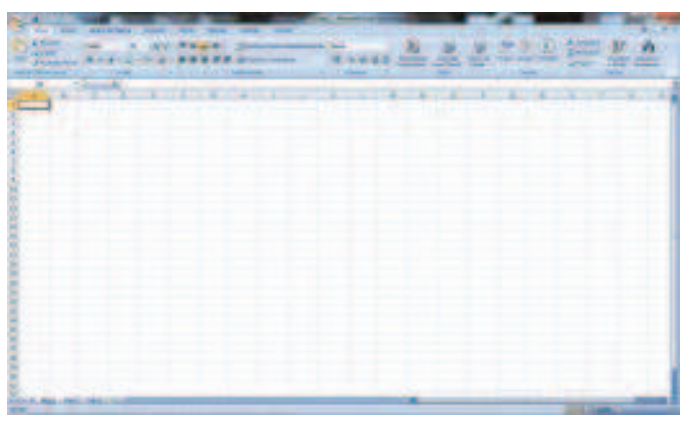

(a) Planilha eletrônica

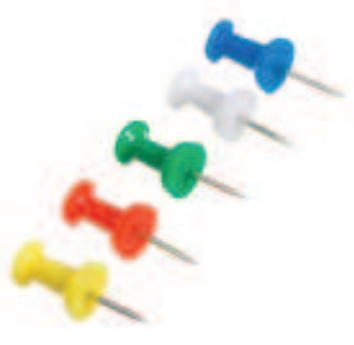

(c) Alfinetes

Para a construção do painel, a função de cada material apresentado na figura 6 é:

- Planilha eletrônica: ferramenta para construção das tabelas para visualização das metas, indicadores e demais informações;

- Post-it: suporte para atualização manual das informações no painel, sendo que a cor do post-it será utilizada como informação também; (b) Post-it

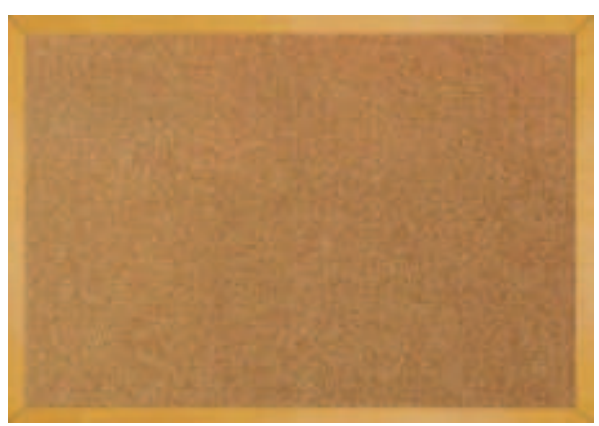

(d) Quadro de rolhas

- Alfinetes: elemento de fixação do post-it e cuja cor será associada aos projetos da faculdade;

- Quadro de rolhas: suporte físico para construção e visualização da Gestão à Vista.

As tabelas elaboradas para os painéis estão apresentadas nas figuras 7, 8, 9 e 10. 
Figura 7: Painel PROJETOS

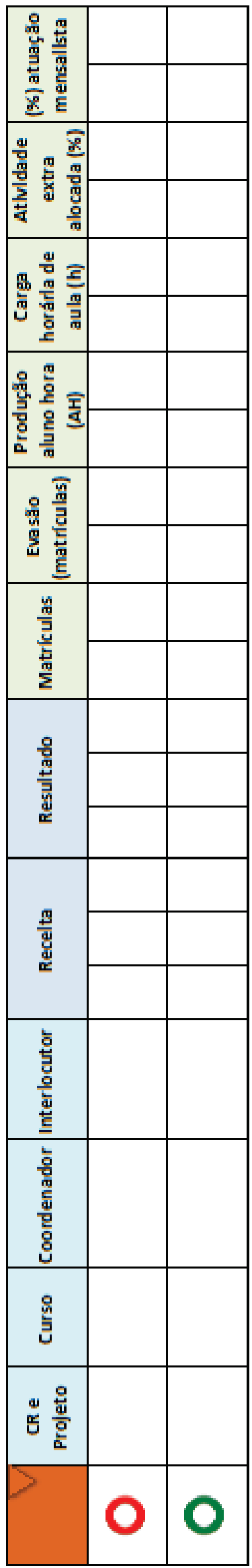

Fonte: Dos autores (2013)
No painel PROJETOS, na primeira coluna, encontra-se a identificação da cor do post-it do mês (no exemplo, laranja) e também da cor do alfinete a ser utilizado em cada projeto. As informações identificadas como relevantes foram organizadas em grupos:

- Identificação-CR/Projeto, nome do curso, Coordenador e Interlocutor;

- Financeira - receita e resultado;

- Produção - matrícula, evasão, aluno-hora, horas de aula e das atividades extra, atuação de mensalistas.

Figura 8: Painel AGENDA CST

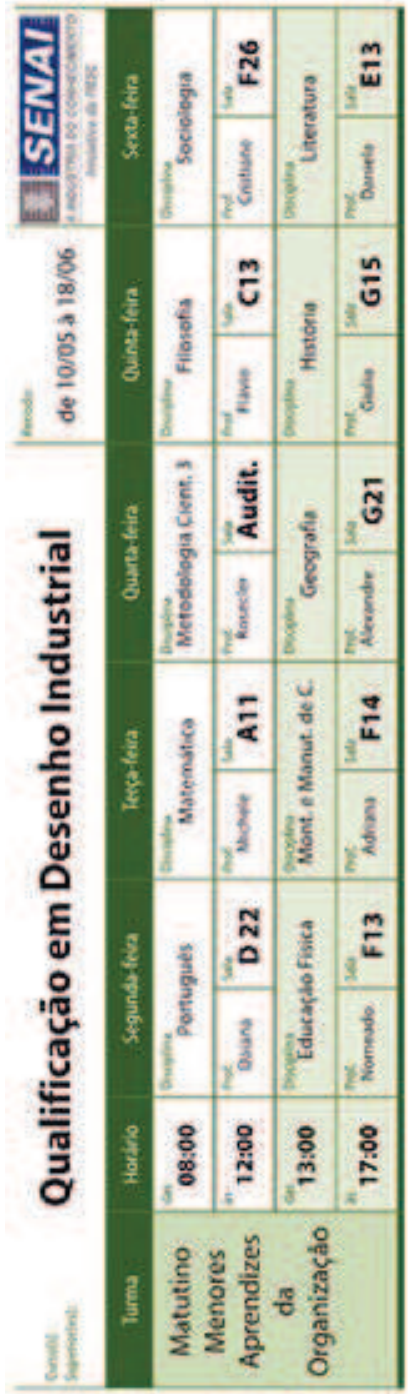

Fonte: Adaptado de SENAI/SC Florianópolis (2013) 
No painel AGENDA CST, utiliza-se de um padrão de horário desenvolvido em meio digital pela faculdade para a disponibilização das seguintes informações: nome do curso, período de vigência do horário, nome da turma, horário das aulas, disciplina (unidade curricular), professor (docente) e sala (ambiente educacional).

Figura 9: Painel AGENDA PÓS

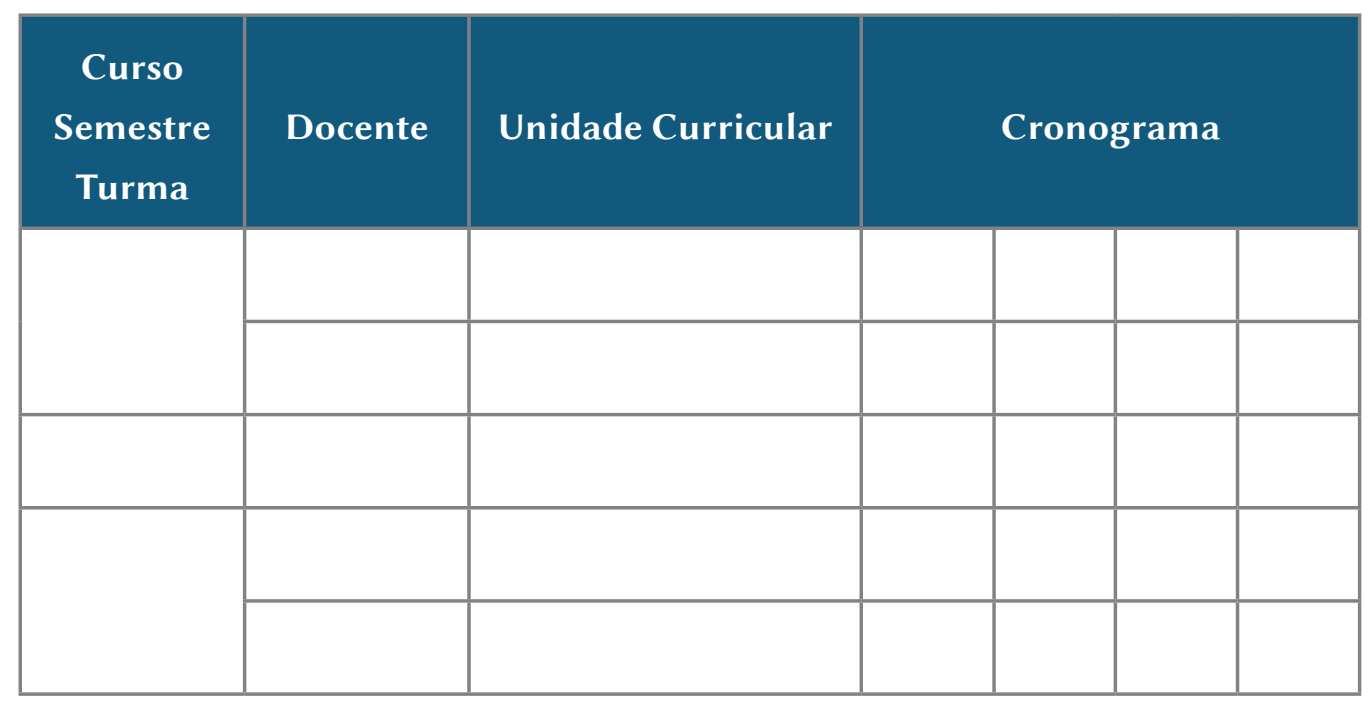

Fonte: Dos autores (2013)

No painel AGENDA PÓS, desenvolveu-se uma tabela com as informações identificadas como relevantes para a modalidade: nome do curso, semestre das unidades curriculares, nome da turma, cronograma.

Figura 10: Painel AGENDA EQUIPE

\begin{tabular}{|c|c|c|c|c|c|c|c|}
\hline Colaborador & Turno & Período & Segunda & Terça & Quarta & Quinta & Sexta \\
\hline & & & & & & & \\
\hline & & & & & & & \\
\hline & & & & & & & \\
\hline & & & & & & & \\
\hline & & & & & & & \\
\hline & & & & & & & \\
\hline
\end{tabular}

Fonte: Dos autores (2013) 
No painel AGENDA EQUIPE, desenvolveuse uma tabela com as informações identificadas como relevantes para a gestão da equipe: nome do colaborador, turnos de trabalho, período de horários dos turnos, dias da semana das atividades.

Vale destacar que, com exceção da AGENDA CST, que pela temporalidade da informação a melhor opção foi o preenchimento em meio digital, todos os outros painéis (PROJETOS, AGENDA PÓS e AGENDA EQUIPE) foram elaborados para serem preenchidos com um post-it padrão $(51 \times 38 \mathrm{~mm})$ inteiro ou cortado no meio.

\subsection{Operacionalização dos Painéis da Gestão à Vista}

A operacionalização do painel e o envolvimento da equipe iniciaram pela construção das tabelas elaboradas, que foram impressas no próprio ambiente de trabalho. A opção ideal é a impressão em formato A3. Todavia, neste trabalho,fez-se a impressão em folhas formato A4. Sugere-se que o painel final seja construído com a participação da equipe, como um mosaico, onde já se inicia a sensibilização e a disseminação da prática com a equipe. A experiência demonstrou que a maior parte das dúvidas foram sanadas dessa maneira e que, inclusive, boas ideias foram identificadas. Entende-se que a primeira versão dos painéis deva ser elaborada pela liderança e discutida num segundo momento com a equipe, primeiramente com os coordenadores e, por último, com os docentes. Sugere-se que a liderança preencha exemplos em cada painel elaborado no primeiro mês da implantação, que realize uma reunião específica para discussão da prática e envie uma mensagem direcionada para as partes interessadas, conforme mensagens padrões apresentadas no Apêndice. O item A.1 do apêndice apresenta uma mensagem que dissemina a Gestão à Vista proposta para toda a equipe.

\subsubsection{Operacionalização do Painel PROJETOS}

O painel PROJETOS tem como objetivo apresentar mensalmente os principais indicadores financeiros e de produção para um acompanhamento de toda a equipe dos projetos, comparando-os com a meta orçamentária do ano e/ou no período.

A atualização é simples e deverá ser feita mensalmente pelos próprios coordenadores de curso, conforme definido nestes passos:

1. buscar os valores financeiros e de produção orçados e realizados, preferencialmente nos relatórios mensais de acompanhamento dos contratos de gestão, ou diretamente nos sistemas corporativos disponíveis (Enterprise Resource Planning - ERP, Intranet).

2. escrever o valor de cada indicador em um post-it padrão e pregá-lo no mural com um alfinete da cor do respectivo projeto, conforme definição in loco na primeira coluna do painel.

3. manter cada indicador atualizado, sendo que na mudança de cada mês do ano, a cor do post-it muda, conforme definição in loco na primeira coluna do painel.

Uma sugestão de comunicação para apresentação do painel de Projetos a todos os coordenadores está apresentada no item A. 2 do apêndice. 


\subsubsection{Operacionalização do Painel AGENDA CST}

O painel AGENDA CST tem como objetivo apresentar semanalmente os horários atualizados dos CSTs para o conhecimento de toda a equipe, possibilitando uma visão atualizada das unidades curriculares, docentes e ambientes utilizados. A atualização será semanale será feita pela publicação interna, dentro do formulário padrão de horários existente, que geralmente já é feito nos murais externos da escola.

O preenchimento é em meio digital e uma sugestão de comunicação deste painel para todos os coordenadores de CSTs está apresentada no item A.3 do apêndice.

\subsubsection{Operacionalização do Painel AGENDA PÓS}

O painel AGENDA PÓS tem como objetivo apresentar mensalmente os horários atualizados das especializações lato sensu para o conhecimento de toda a equipe, possibilitando uma visão atualizada das unidades curriculares, docentes e cronograma de realização. A atualização será, no mínimo, mensal e feita pelo preenchimento das seguintes informações:

1. buscar o nome oficial do curso no painel PROJETOS e preencher um post-it na cor verde, pregando-o como respectivo alfinete da cor do projeto;

2. escrevero semestre das unidades curriculares em um post-it de cor amarela, pregando-o com o respectivo alfinete da cor do projeto;

3. escrever o nome da turma em um post-it na cor laranja, pregando-o com o respectivo alfinete da cor do projeto;

4. escrever as datas de cada encontro presencial das UCs em um post-it na cor amarela, pregando-o com o respectivo alfinete da cor do projeto.

Uma sugestão de comunicação deste painel para todos coordenadores de pós está apresentada no item A.4 do apêndice.

\subsubsection{Operacionalização do Painel AGENDA EQUIPE}

O painel AGENDA EQUIPE tem como objetivo apresentar semanalmente os horários atualizados da agenda de todos, bem como conhecer as atividades e os projetos nos quais estes estão envolvidos e as áreas de conhecimento de cada atuação. A atualização será feita pela publicação de uma agenda semanal das atividades de cada membro da equipe, lembrando que cada um será responsável pela atualização de sua própria agenda.

Uma sugestão de preenchimento deve seguir estas diretrizes:

1 - buscar as informações do ensalamento ${ }^{1}$ com seu coordenador de referência e sobre as atividades previstas para o semestre;

2 - identificar a cor do post-it de cada atividade, verificando a legenda disponível in loco;

3 - escrever informações básicas de cada atividade nos post-it desta forma:

3.1 - se for tipo [Aula], o nome da UC;

3.2 - se for tipo [Preparação de aula], o(s) nome(s) da(s) UC(s);

3.3 - se for tipo [Coordenação], o nome do curso;

\footnotetext{
${ }^{1}$ Ensalamento é a denominação no SENAI/SC para o processo de construção dos horários dos cursos de EPT, onde são definidos para cada horário nos dias letivos do ano: a UC, $\mathrm{o}$ ambiente de ensino e o respectivo docente. $\mathrm{O}$ processo de ensalamento leva em consideração a carga horária de cada UC, a disponibilidade dos ambientes e docentes e deve ser implementado levando em consideração as métricas orçamentárias em vigor no ano letivo de sua implementação.
} 
3.4 - se for tipo [Orientação de TCC], o nome da modalidade;

3.5 - se for tipo [Olimpíada do Conhecimento], o nome da modalidade;

3.6 - se for tipo [Projeto de Curso], o nome do projeto ou modalidade;

3.7 - se for tipo [STT], o nome do projeto.

4 - observar que um post-itinteiro representa um turno,logo, meio post-it representa meio turno; 5 - cuidar para usar o alfinete da cor correta do projeto para pregar os post-its.

Uma sugestão de comunicação deste painel para toda equipe está apresentada no item A.5 do apêndice.

\subsection{Primeiros Resultados}

Os painéis de Gestão à Vista foram implantados e estão em fase de preenchimento, conforme pode ser visto na figura 11.

Figura 11: Proposta de Gestão à Vista

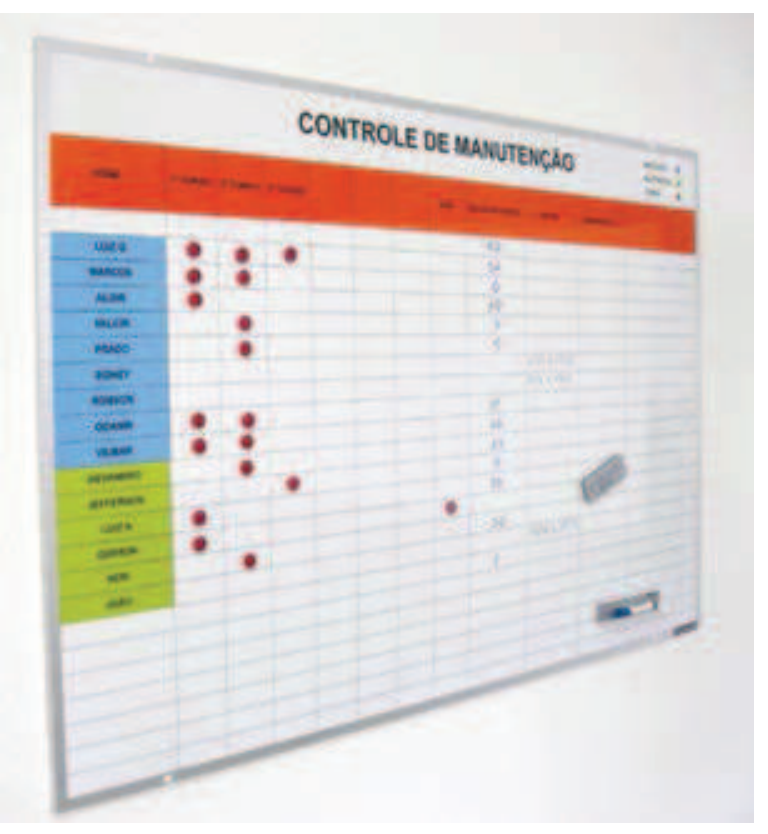

Fonte: Dos autores (2013)
Como primeiros resultados obtidos, percebeuse um aumento do conhecimento de toda equipe dos projetos existentes na Faculdade, em que o painel PROJETOS passou a ser a referência da comunicação dos indicadores do negócio.

Percebeu-Se Que, COM O CO-

NHECIMENTO AMPLIADO DOS PRO-

JETOS, SUA CONTRIBUIÇÃO PARA

O ORÇAMENTO E DOS PRINCIPAIS

INDICADORES, QUE AS DISCUSSÕES

NAS REUNIÕES PERIÓDICAS COM A

EQUIPE TENDERAM PARA A SOLU-

ÇÃO DOS PROBLEMAS DE FORMA

MAIS COLABORATIVA.

Da mesma forma, com o conhecimento das agendas dos cursos e das pessoas, tem-se uma visão sistêmica dos produtos em execução, bem como dos recursos da faculdade, tanto da equipe como dos ambientes educacionais. 


\section{CONCLUSÃO}

Com base nos objetivos definidos, conclui-se que, com relação à identificação das necessidades de práticas de gestão à vista aplicáveis à EPT, foram identificadas as necessidades essenciais na gestão de uma faculdade. Com os indicadores financeiros e de produção dos projetos, foi possível o acompanhamento do orçado e realizado nestes projetos. $\mathrm{E}$ com o conhecimento das agendas dos cursos e da equipe, promoveu a identificação dos recursos disponíveis e das lacunas existentes.

Com relação à elaboração de uma proposta de mural, com painéis de gestão à vista para uma instituição de EPT, projetou-se um mural composto de painéis e materiais acessíveis, que possibilitam replicabilidade e permitem customizações conforme as peculiaridades encontradas nas instituições. A proposição foi direcionada para uma faculdade de tecnologia que possui as modalidades de curso superior de tecnologia e pós lato sensu.

Sobre implantação da proposta de mural de gestão à vista em uma faculdade de tecnologia do SENAI/SC, ao implantar e promover a operacionalização do painel, percebeu-se uma ampliação no conhecimento e no envolvimento da equipe, principalmente para os docentes, que não tinham uma visão sistêmica da faculdade. Percebeu-se, também, um aumento no compartilhamento de boas práticas entre os coordenadores, independente da modalidade.

Para que a gestão à vista funcione efetivamente, o método de PDCA é essencial e deve ser usado em conjunto, pois este será a ferramenta de suporte para interpretação dos indicadores e proposição de ações que direcionam ao alcanlce das metas orçamentárias.

Identifica-se como trabalho futuro, o desenvolvimento da gestão à vista nas outras modalidades da EPT: Qualificação Profissional Básica (QPB),Aprendizagem Industrial(AI) e Cursos Técnicos (CT). Sugere-se, ainda, que desenvolva-se um trabalho nas modalidades ligadas à Tecnologia e Inovação: Serviços Técnicos e Tecnológicos e Pesquisa Aplicada. 


\section{Visual Management in Practice in Vocational and TECHNOLOGY EDUCATION: AN application in the College OF TECHNOLOGY SENAI / SC FLORIANÓPOLIS}

\begin{abstract}
Communication is essential in business and is one of the mainfactors that promote thenecessaryconditions forleadership. Within thiscontext, when well developed, visual management is an effective and efficient communication practice. This article aims at proposing the implementation of the visual management in an institution for Professional Education and Technology (EPT) for the identification ofneeds, development andimplementation of a proposed visual management mural in a of technology Faculty of SENAI / SC. The developed theoretical framework starts with the strategic management with a view to the results through goals, passes through the management tool PDCA (Plan-Do-Check-Act) and ends with the visual management approach advocated by the Toyota Production System. Methodologically, this work is of an applied nature, with qualitative and quantitative approach, with exploratory objective, baving an experimentalcharacter, with confirmation based upon an action-research activity. The steps developed in the methodology were planning, development and operationalization of the panel, the evaluation of the implementation. As firstresults, it wasnoticedanincrease in the knowledge of all staff in the Faculty of existing projects, assuming a greater motivation to solve problems that arise in these projects. Likewise, with the knowledge of the agendas of courses and people, we have a systemic vision of the Faculty resources, both from staff and educational environments points of view. We conclude that the essential needs in the management of a Faculty were identified, that the proposition of the mural adapts to a Faculty of technology and also we noticed an increase in the sharing of good practices among course coordinators.
\end{abstract}

Keywords: Visual

Management. Vocational and

Technical Education. Goals.

Indicators.

\section{REFERÊNCIAS}

BSI. Balanced Scorecard Institute. Disponível em: <http://www.balancedscorecard.org/ BSCResources/AbouttheBalancedScorecard/ tabid/55/Default.aspx>. Acesso em: 16 ago. 2013.

FALCONI, V. O verdadeiro poder. Nova Lima: INDG Tecnologia e Serviços Ltda., 2009.

GIL, A. C. Como Elaborar Projetos de Pesquisa. São Paulo: Atlas, 2002.

JONES, D. Gerenciando Visualmente. 2011. Disponível em: <http://www.lean.org.br/ comunidade/artigos/pdf/artigo154.pdf $>$. Acesso em: 16 ago. 2013.

KAPLAN, R. S. NORTON, D. P. A estratégia em ação: balanced scorecard. Rio de Janeiro: Editora Campus, 1997.

Mapas estratégicos: convertendo ativos intangíveis em resultados tangíveis. Rio de Janeiro: Elsevier, 2004.

. Organização orientada para a estratégia. Rio de Janeiro: Editora Campus, 2000.

LIB. Lean Institute Brasil. 2013. Disponível em: $<$ http://www.lean.org.br/>. Acesso em: 16 ago. 2013.

SEVERINO, A. J. Metodologia do Trabalho Científico. São Paulo: Cortez, 2007.

SHOOK, J. A gestão visual - a boa, a ruim e a feia. 2012. Disponível em: <http://www.lean.org. br/comunidade/artigos/pdf/artigo192.pdf >. Acesso em: 16 ago. 2013.

SILVA, E. L.; MENEZES, E. M. Metodologia da pesquisa e elaboração de dissertação. Florianópolis: UFSC, 2005.

SOBEK, D. K. SMALLEY, A. Entendendo o Pensamento A3. Porto Alegre: Bookman, 2010.

TANAKA, S. Lean for Knowledge Work. LeanSummit 2010, UK: nov. 2010. Disponível em: <http://www.leanuk.org/pages/ eventsummit2010speakertanaka.htm >. Acesso em: 16 ago. 2013.

TANNER, S. Lean for Leaders in the Executive Office. LeanSummit 2010, UK: nov. 2010.

Disponível em: <http://www.leanuk.org/pages/ eventsummit2010speakertanner.htm>. Acesso em: 16 ago. 2013.

Data de recebimento: $10 / 01 / 14$

Data de aprovação: 29/10/14 


\section{APÊNDICE}

\section{A.1 - Proposição de mensagem para apresentação da Gestão à Vista}

ASSUNTO: Gestão à Vista - visão geral

\section{SUGESTÃO DE TEXTO:}

Prezada Equipe!

Boa tarde!

Proponho implementarmos uma gestão à vista no nosso ambiente com o objetivo de facilitar a comunicação dos principais aspectos da gestão em um núcleo de negócio em EPT no SENAI/SC. As premissas utilizadas neste projeto foram: simplicidade, utilidade, flexibilidade, alfinetes e post-its.

Cada membro da equipe terá uma função de atuação neste modo de gestão, conforme os painéis que foram elaborados:

1 - PROJETOS - principais indicadores dos projetos do núcleo - responsabilidade de atualização: todos os coordenadores - frequência: mensal.

2 - AGENDA CST - horários dos cursos superiores de tecnologia (CST) - responsabilidade de atualização: coordenadores CST - frequência: semanal.

3 - AGENDAPÓS - cronograma das aulas de pós - responsabilidade de atualização: coordenadores $\underline{P O ́ S}$ - frequência: $\underline{\text { mensal. }}$.

4 - AGENDA EQUIPE - ocupação semanal da equipe do núcleo - responsabilidade de atualização: todos - frequência: semanal.

Para cada painel serão enviadas mensagens específicas para a utilização dos respectivos responsáveis.

Com a fluência da comunicação, entende-se que todos tendem a ajudar na solução dos problemas inerentes ao nosso negócio.

Conto com a usual colaboração e envolvimento de todos!

E, claro, sugestões/criticas/melhorias serão sempre bem-vindas!

Abraço e obrigado,

\section{ASSINATURA DO GESTOR}




\section{A.2 - Proposição de mensagem para apresentação do Painel PROJETOS}

ASSUNTO: Gestão do Orçamento à Vista - Painel PROJETOS

\section{SUGESTÃO DE TEXTO:}

\section{Prezados Coordenadores!}

Boa tarde!

O painel PROJETOS tem como objetivo apresentar mensalmente os principais indicadores financeirose de produção para um acompanhamento de toda a equipe dos nossos projetos, comparando-os com a meta orçamentária do ano.

A atualização será mensal e é muito simples, basta seguir estes passos:

1 - buscar os valores preferencialmente nos relatórios dos contratos de gestão (realizado) na nossa rede interna, ou diretamente nos sistemas corporativos (Benner, SGN, relatórios da Intranet).

2 - escrever o valor de cada indicador num post-it padrão $(51 \times 38 \mathrm{~mm})$, dobrando-o ao meio e pregando-o no mural com um alfinete da cor do seu projeto (vide legenda in loco) na respectiva linha deste; 3 - manter cada indicador atualizado, sendo que na mudança de cada mês do ano, a cor do post-it muda (nos dados consolidados de maio a cor é amarela).

Vamos pôr a mão na massa epreencher os indicadores de maio? Alguns como exemplo já foram preenchidos.

Com o conhecimento dos principais indicadores, entende-se que todos tendem a ajudar na solução dos problemas que surjam nos projetos.

Conto com a usual colaboração e envolvimento de todos!

E, claro, sugestões/criticas/melhorias serão sempre bem-vindas!

Abraço e obrigado,

\section{ASSINATURA DO GESTOR}




\section{A.3 - Proposição de mensagem para apresentação do Painel AGENDA CST}

ASSUNTO: Gestão dos horários à Vista - Painel AGENDA CST

\section{SUGESTÃO DE TEXTO:}

\section{Prezados Coordenadores CST!}

Boa tarde!

O painel AGENDA CST tem como objetivo apresentar semanalmente os horários atualizados dos nossos CSTs para o conhecimento de toda a equipe, possibilitando ter uma visão atualizada das unidades curriculares (UCs), docentes e ambientes utilizados.

A atualização será semanal e será feita pela publicação interna, dentro do template padrão de horários, do que já é feito nos murais da Faculdade das seguintes informações:

1 - nome do curso;

2 - periodo de vigência do horário;

3 - nome da turma;

4 - borário das aulas;

5 - disciplina $(U C)$;

6 - professor (docente);

7 - $\underline{\text { sala }}$ (ambiente educacional).

A agenda do CST Análise e Desenvolvimento de Sistemas já está no mural como exemplo.

Com o conhecimento dos horários, entende-se que todos tendem a ajudar na solução das principais dúvidas dos alunos e docentes:

- Onde está a minha turma? Qual é a sala mesmo?

- Quem é o meu professor?

- Quais as UCs de hoje?

Conto com a usual colaboração e envolvimento de todos!

E, claro, sugestões/criticas/melhorias serão sempre bem-vindas!

Abraço e obrigado,

\section{ASSINATURA DO GESTOR}




\section{A.4 - Proposição de mensagem para apresentação do Painel AGENDA PÓS}

ASSUNTO: Gestão dos horários à Vista - Painel AGENDA PÓS

\section{SUGESTÃO DE TEXTO:}

Prezados Coordenadores de PÓS!

Boa tarde!

O painel AGENDA PÓS tem como objetivo apresentar mensalmente os horários atualizados das nossas especializações lato sensu para o conhecimento de toda a equipe, possibilitando ter uma visão atualizada das unidades curriculares (UCs), docentes e cronograma de realização.

A atualização será no minimo mensal e feita pelo preenchimento das seguintes informações:

1 - nome do curso - buscar o nome oficial no painel PROJETOS e preencher um post-it inteiro na cor verde, pregando-o com o respectivo alfinete do seu projeto (vide painel PROJETOS);

2 - semestre das unidades curriculares - escrever o semestre em um meio post-itna cor amarela, pregando-o com o respectivo alfinete do seu projeto (vide painel PROJETOS);

3 - nome da turma - escrever o nome da turma em um post-it inteiro na cor laranja, pregando-o com o respectivo alfinete do seu projeto (vide painel PROJETOS);

4 - cronograma - escrever as datas de cada encontro presencial das UCs em meio post-it na cor amarela, pregando-o com o respectivo alfinete do seu projeto (vide painel PROJETOS).

Vamos pôr a mão na massa e preencher as informaçôes das turmas de pós em andamento? Verifiquem o exemplo da pós em Engenharia de Software presencial já inserida no painel.

Com o conhecimento prévio dos cronogramas, podemos antecipadamente trabalhar lacunas de docentes ou de datas que venham a surgir nos nossos projetos.

Conto com a usual colaboração e envolvimento de todos!

E, claro, sugestões/criticas/melhorias serão sempre bem-vindas!

Abraço e obrigado,

\section{ASSINATURA DO GESTOR}




\section{A.5 - Proposição de mensagem para apresentação do Painel AGENDA EQUIPE}

ASSUNTO: Gestão dos horários à Vista - Painel AGENDA EQUIPE

\section{SUGESTÃO DETEXTO:}

Prezada Equipe!

Boa tarde!

Opainel AGENDAEQUIPE tem como objetivo apresentar semanalmente os horários atualizados da agenda de todos, bem como conhecer as atividades e os projetos nos quais estão envolvidos, e áreas de conhecimento de atuação.

A atualização será feita pela publicação de uma agenda semanal das atividades de cada membro da equipe. Lembrando que cada um é responsável pela atualização da sua agenda.

O preenchimento é muito simples, basta seguir estas diretrizes:

1 - buscar na planilha de ensalamento ou com seu coordenador de referência, quais as atividades previstas para o semestre (no caso vamos desenvolver para o semestre 2013.2);

2 - identificar a cor do post-it de cada atividade, verificando a legenda disponivel in loco;

3 - escrever informaçôes básicas de cada atividade nos post-it dessa forma:

3.1 - se for tipo [Aula], o nome da UC;

3.2 - se for tipo [Preparação de aula], o(s) nome(s) da(s) UC(s);

3.3 - se for tipo [Coordenação], o nome do curso;

3.4 - se for tipo [Orientação de TCC], o nome da modalidade;

3.5 - se for tipo [Olimpiada do Conhecimento], o nome da modalidade;

3.6 - se for tipo [Projeto de Curso], o nome do projeto ou modalidade; e

3.7 - se for tipo [STT], o nome do projeto.

4-observar que 1post-it inteiro representa 1 turno, logo meio post-it representa meio turno;

5 - cuidar para usar o alfinete da cor do seu projeto (vide painel PROJETOS) para pregar os seus post-its.

Vamos pôr a mão na massa e preencher as nossas agendas para 2013.2? Alguns exemplos da equipe já foram postados neste painel.

Com o conhecimento de todos das nossas atividades, entende-se que reforçaremos o conhecimento da atuação da equipe e saberemos quem poderá nos ajudar na solução dos eventuais problemas que surjam nos projetos.

Conto com a usual colaboração e envolvimento de todos!

E, claro, sugestöes/criticas/melhorias serão sempre bem-vindas!

Abraço e obrigado,

\section{ASSINATURA DO GESTOR}




\section{SOBRE OS AUTORES}

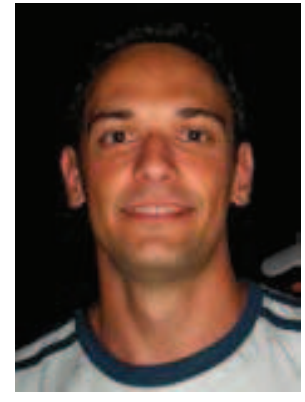

\section{Juliano Anderson \\ Pacheco}

Engenheiro Eletricista graduado pela Universidade Federal de Santa Catarina (UFSC), mestre em Ciências da Computação, ênfase em Estatística,e Doutorem Engenharia de Produção,ênfase em Inteligência Organizacional, ambos também pelaUFSC.Atuou como Professore Coordenadorde Curso Superior de Tecnologia, como Coordenador do Núcleo de Tecnologia da Informação e como Coordenador da Faculdade de Tecnologia (CTAI) do SENAI/SC em Florianópolis.Atua como Consultor Interno do Sistema FIESC, na Coordenadoria de Planejamento e Controle da Gestão (COPLAC). Tem experiência nas áreas de Telecomunicações, Ciência da Computação,Estatística,Administração e Marketing, com ênfase em Redes Convergentes, Sistemas deInformação,Análise Estatística de Dados e Geoprocessamento, Planejamento Estratégico, Prospectiva Estratégica, Roadmapping, Planos de Negócios e Gestão Educacional.

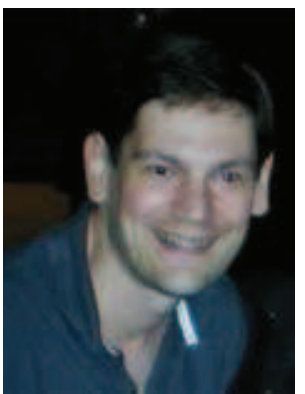

\section{Maycon Cim}

Possui Certificação PMP, é Especialista em Gerenciamento de Projetos pelo SENAI/ Florianópolis, Tecnólogo em Gerenciamento dos Sistemas daInformação,pelo SENAC/Florianópolis, Técnico em Processamento de Dados pelo SENAC/ Brusque. Experiência em docência na área de Tecnologia da Informação e Gestão de Projetos. Autor de livros didáticos na área de Desenvolvimento para web, Excel Avançado e Gestão de Projetos. Possui vasta experiência na prática de gestão de projetos, liderança de equipes, gestão visuale comércio eletrônico. Atualmente trabalha no SENAI/SC em Florianópolis como Coordenador do Núcleo de Cursos Técnicos e Assessoria e Consultoria. 